\title{
APPLICATION OF THE RAIL VEHICLE'S MONITORING SYSTEM IN THE PROCESS OF SUSPENSION CONDITION ASSESSMENT
}

Rail vehicle's suspension determines riding dynamics, safety and comfort. Its condition should be checked regularly, and even monitored continuously. In order to acquire information on suspension condition during commercial operation, the rail vehicles' monitoring system has been developed at Monitoring of Technical State of Construction and Evaluation of its Lifespan Project (MONIT). The aim of the system is qualitative assessment of suspension condition based on acceleration signals recorded on bogie frames and body.

The experimental tests of the system were performed on wagons with introduced suspension damages: stiffness reduction of primary suspension in freight car and the loss of damper of secondary suspension in passenger car. The monitoring procedure and test results are presented in the work.

Keywords: Suspension, condition monitoring, fault detection.

\section{Introduction}

Currently used monitoring systems of the rail vehicles are directly related to the control of the vehicle, e.g. power supply, power train, braking system. Rail vehicles' suspension has not been an object of the monitoring although it determines significantly riding dynamics, safety and comfort. The rail vehicle monitoring system has been developed at Monitoring of Technical State of Construction and Evaluation of its Lifespan Project (MONIT). The main assumption for the system is qualitative suspension condition assessment by means of standard measuring equipment during normal operation.

The system has been tested on wagons with and without suspension damages. Recorded data during the test provided information on the vehicle dynamic behavior, affected by suspension faults.

\section{Monitoring system}

Expedient feature of the monitoring system of rail vehicle and track is versatility and modular architecture. It can be installed virtually on every type of rail vehicle. Its aim is qualitative assessment of the primary and secondary suspension, axle bearing temperature measurement and evaluation of track quality.

The system consists of:

- Central data acquisition computer MOXA V2406-XPE,

- Sierra Wireless AirLink GX400 router with GPS module,

- Local data acquisition units,

- Piezoelectric VIS-311A accelerometers,

- Pt100 temperature sensors,

- System server with operator's station.

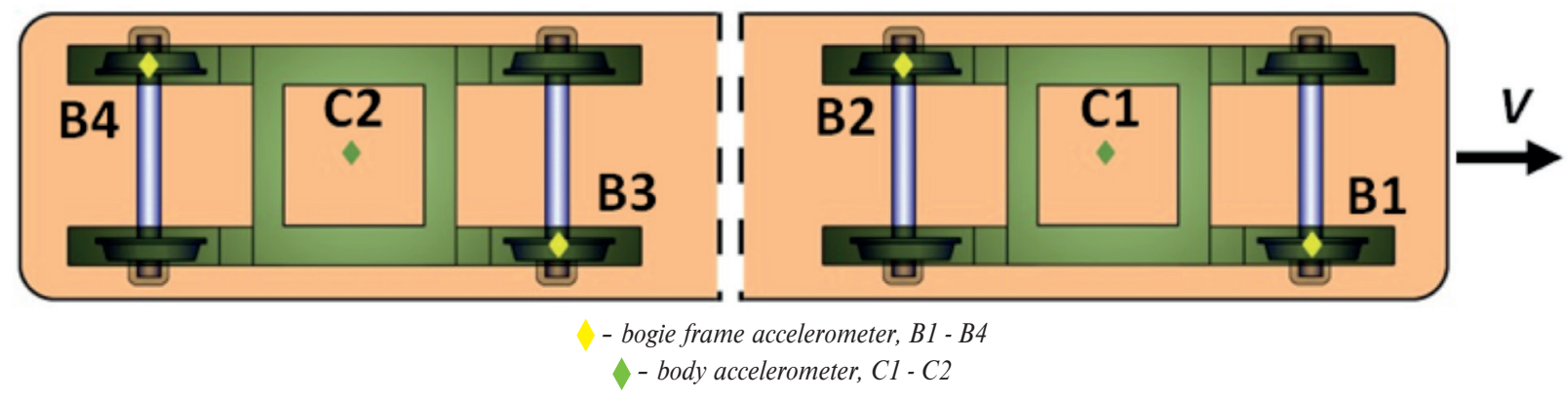

Fig. 1 The location of measurement points

\footnotetext{
* Rafal Melnik, Bogdan Sowinski

Warsaw University of Technology, Faculty of Transport, Department of Transport Equipment Construction Bases, Warsaw, Poland, E-mail: rme@wt.pw.edu.pl
} 
Location of measurement points is depicted in Fig 1. Acceleration signals recorded on a bogie frame above wheel sets (B1-B4) are used for primary suspension condition assessment. Regarding the system's architecture and cost of the equipment the number of measurement points is reduced to two. This solution is still sufficient for qualitative assessment. Sensors installed on a body, above bogie center $(\mathrm{C} 1, \mathrm{C} 2)$ are used in order to monitor secondary suspension condition. In each measurement point acceleration signals are recorded in vertical $(Z)$ and lateral (Y) direction.

Length of a track section, on which monitoring system performs measurement, is adjustable and is set to 500 meters. The signals from all sensors are collected in the form of a data packet. This packet contains data re-sampled to spatial frequency $\mathrm{fs}=5 \mathrm{~m}-1$ which were previously analyzed. The data packet contains geographic coordinates of the place where the signals recording was triggered, and values of the signals. Exceedance of the diagnostic indicator limit value results in generation of a control packet containing warnings or alerts (depending on the exceedance value). Packets are sent to the operator's station (system's server) via GSM network where information occurs on warnings and alerts referring to the vehicle's condition. System enables vehicle localization by means of GPS module.

\section{Suspension condition monitoring process}

Suspension fault detection method for the monitoring system should be efficient, easy to implement and fast. There are a few methods, e.g. based on Kalman Filter [1 and 2] and multimodel approach [3 and 4] which are intended to solve the problem of suspension fault detection. These methods require mathematical model of the vehicle whose parameters: masses, moments of inertia, stiffness and damping coefficients may not be available. The proposed fault detection method does not require mathematical model since for qualitative assessment of suspension condition the analysis of the signals is incorporated.

The procedure, in terms of the running gear's dynamic properties and safety, is based on the requirements contained in UIC 518 leaflet [5] and in the standard EN 14363 [6]. The limit values of RMS and percentile 99.85\% (maximal value) of acceleration signals, measured in specified points of vehicle, should not be exceeded.

The influence of suspension damages on acceleration signals is rather complex. The effect of stiffness or damping reduction on acceleration signals depends generally on the magnitude and frequency of the excitation (track geometrical irregularities, vehicle speed). Regarding damaged suspension, in certain circumstances the registered values of acceleration may be lower compared with a vehicle in undamaged condition. However, the change of speed or track section can result in the increase of the acceleration values for the damaged vehicle.

The normative parameters may not be sufficient for damage detection, therefore, monitoring procedure involves additional statistical parameters, which are not included in [5] and [6]. Various statistical parameters have been considered for this problem and it was concluded that the diagnostic process is more efficient when a set of parameters is used [7]. Finally, the set consisting of RMS, Interquartile range and Zero-Peak were chosen for the diagnostic process.

In papers [7, 8 and 9] there was a lack of algorithm that could link together all analyzed diagnostic parameters. Consideration of these parameters separately requires assigning limit values for each of them. In order to improve fault detection and to treat these parameters as one diagnostic indicator a multidimensional analysis is proposed.

The basic assumptions of multidimensional approach are listed below:

- Selected statistical parameters providing diagnostic information can be used in creation of multidimensional space,

- A point in multidimensional state space is defined by coordinates whose values correspond to the values of diagnostic parameters,

- The point representing a vehicle's faulty condition is distant from the point of a vehicle's nominal condition,

- The excess of certain (established) distance is a diagnostic symptom.

The current condition (at present time) of the vehicle is characterized by the parameters of acceleration signals recorded on a certain distance on a track of known maintenance quality.

For the vehicle in nominal condition (without suspension damages) we can obtain many Niv (1) points belonging to the diagnostic space. Each of these points refers to i-th passage on the same track section of known maintenance quality, at the same velocity v.

$$
N_{i}^{v}=\left(x_{N 1, i}^{v}, x_{N 2, i}^{v}, \ldots, x_{N n, i}^{v}\right)
$$

where,

$N_{i v}^{i}$ - a point referring to current condition at velocity v of coordinates $x_{N 1, i}^{v}, x_{N 2, i}^{v}, \ldots, x_{N n, i}^{v}$,

$i$ - the number of measurement,

$x_{N 1, i}^{v}, x_{N 2, i}^{v}, \ldots, x_{N n, i}^{v}$ - the following values of diagnostic parameters for the current state from 1 to n, for velocity v.

The reference point $S^{v}(2)$ is created by averaging the coordinates (diagnostic parameters' values) of many $N_{i}^{v}$ points. This point represents a reference vehicle - vehicle in nominal condition which parameters' values are mean values obtained from many vehicle passages.

$$
S^{v}=\frac{1}{n} \sum_{i=1}^{n} N_{i}^{v}
$$

where,

$x_{S 1}^{v}, x_{S 2}^{v}, \ldots, x_{S n}^{v}$ - the following values of the coordinates from $1 \mathrm{do} \mathrm{n}$ for the reference point and velocity $\mathrm{v}$.

The point representing current state $U_{j}^{v}$ (3) is described by coordinates (parameters) values of the signals recorded during $\mathrm{j}$-th measurement during normal operation, on the same track section as for $N_{i}^{v}$ point and at the same speed.

$$
U_{j}^{v}=\left(x_{U 1, j}^{v}, x_{U 2, j}^{v}, \ldots, x_{U n, j}^{v}\right)
$$


where,

$U_{j}^{v}$ - a point representing current condition at velocity $\mathrm{v}$ of coordinates $x_{U 1, j}^{v}, x_{U 2, j}^{v}, \ldots, x_{U n, j}^{v}$,

$j$ - the number of measurement,

$x_{U 1, j}^{v}, x_{U 2, j}^{v}, \ldots, x_{U n, j}^{v}$ - the following values of diagnostic parameters from $l$ to $n$, velocity $v$.

Since three parameters were selected for diagnostic process, we get $i, j=3$, a three-dimensional diagnostic space.

The fault detection of running gear, especially suspension, is based on the method of analysis of metric (distance) $d\left(S^{v}, U_{j}^{v}\right)$ from the reference point and point representing current state. The concept of the method is illustrated in Fig. 2. The diagnostic symptom is the excess of the permissible distance $F$ from the reference point. The $\mathrm{F}$ value may be set upon results obtained from experiments in which suspension damages were studied. The distance can be calculated using different formulas, albeit Euclidean metric is used.

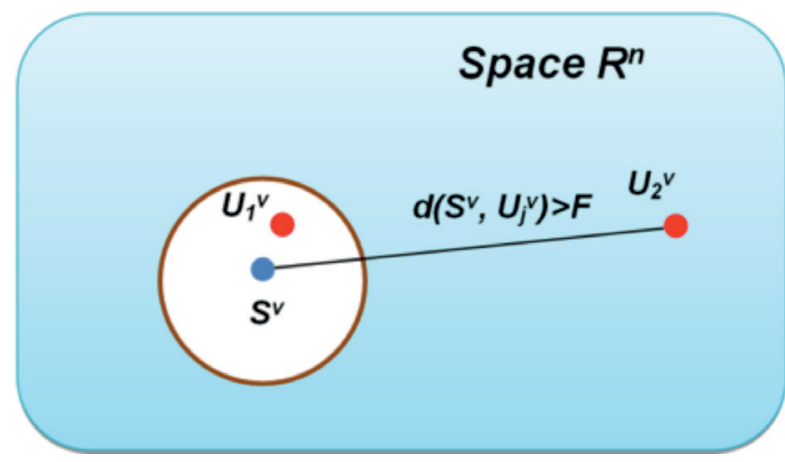

Fig. 2 The concept of the method

The points referring to current condition should be located within boundaries defined by $\mathrm{F}$, of the reference point $S^{v}$. It is not crucial on which side of $S^{v}$ the $U_{j}^{v}$ point is located. This allows using this method in detection of faults that result in the increase and/or decrease of the parameters values. Since the range of values for the following parameters may be different, they should be normalized by dividing values of $U_{j}^{v}$ by values of $S^{v}$, therefore, the reference points coordinates are $(1,1,1)$.

\section{Experimental tests}

The aims of the experimental tests were validation of the monitoring system and studies of the influence of suspension damages on recorded signals. The monitoring system was installed on a passenger and freight car (equipped with Y25 bogies). The freight car was loaded up to $90 \mathrm{t}$. For validation purpose an additional measuring system was installed, which is used by the Railway Institute (Poland) for approval tests. The experiment took place on a test track in Zmigrod in Poland. The first stage of the experiment was carried out on vehicles in nominal condition in order to obtain reference signals. In the second stage, the test was performed on wagons with introduced suspension damages:
- passenger car - removal of one secondary suspension damper (Fig. 3),

- freight car - removal of one coaxial springs packet of secondary suspension, on the left side of axle bearing at one wheel (Fig. 4).

To minimize the risk of accident or even derailment, only one sort of damage was introduced during the test ride.

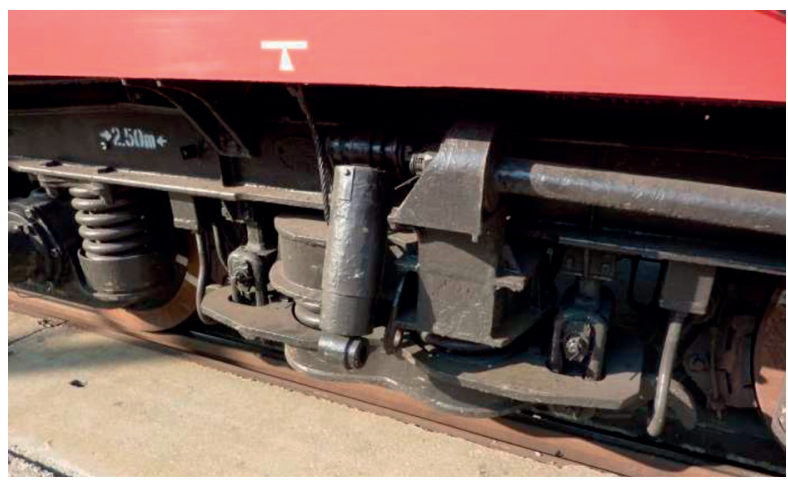

Fig. 3 Passenger car running gear with secondary suspension damper disconnected

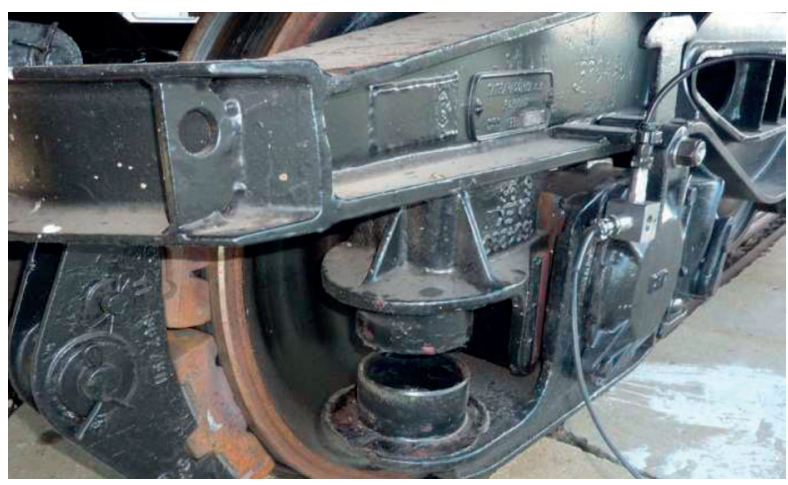

Fig. 4 Freight car bogie with removed springs on one side

\section{Results of the experiment}

The acceleration signals were analyzed using the proposed method. The number of markers in plots (points in Figs. 5 - 8) depends on the number of passages on chosen track sections. The $F$ value (radius of the sphere) equals to standard deviation of the distances' value (of nominal vehicle) from the reference point and is calculated for different speed values and track sections. For commercial use it may be adjusted directly on the results of the experiment. Since the axes are not equal in Figs. $5-8$, the sphere appears as an ellipsoid.

The case of damaged passenger car (damping reduction) condition is illustrated in Figs. 5 and 6. The blue star marker represents the reference point, blue point - condition assessed as undamaged, red point - condition assessed as damaged. The coordinates' normalized values are presented below plots. Vertical acceleration signals from four passages on the same tangent track section were used for condition evaluation. In these figures it can 
be seen that not every point referring to a vehicle with suspension fault is classified as damaged. Nevertheless, for some passages (measurements) distances are substantial and faulty condition can be identified. For $80 \mathrm{~km} / \mathrm{h}$ and vertical direction $\mathrm{F}=0.48$ and $\mathrm{F}=0.232$ for $100 \mathrm{~km} / \mathrm{h}$.

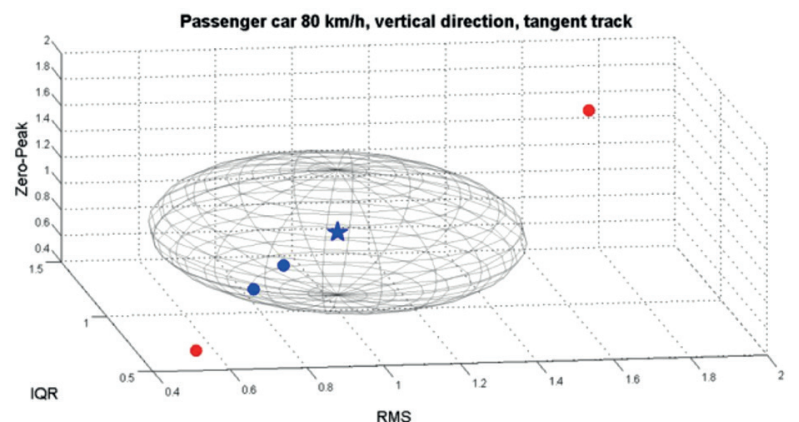

\begin{tabular}{|c|c|c|c|}
\hline RMS $(\mathrm{Z})$ & $\mathrm{IQR}(\mathrm{Z})$ & Zero-Peak $(\mathrm{Z})$ & $\mathrm{d}(\mathrm{Sv}, \mathrm{Ujv})$ \\
\hline 0.744 & 0.844 & 0.715 & 0.413 \\
\hline 1.689 & 1.147 & 1.756 & 1.033 \\
\hline 0.822 & 0.844 & 0.892 & 0.260 \\
\hline 0.533 & 0.587 & 0.478 & 0.813 \\
\hline
\end{tabular}

Fig. 5 Results of passenger car analysis, $80 \mathrm{~km} / \mathrm{h}$, vertical acceleration, tangent track

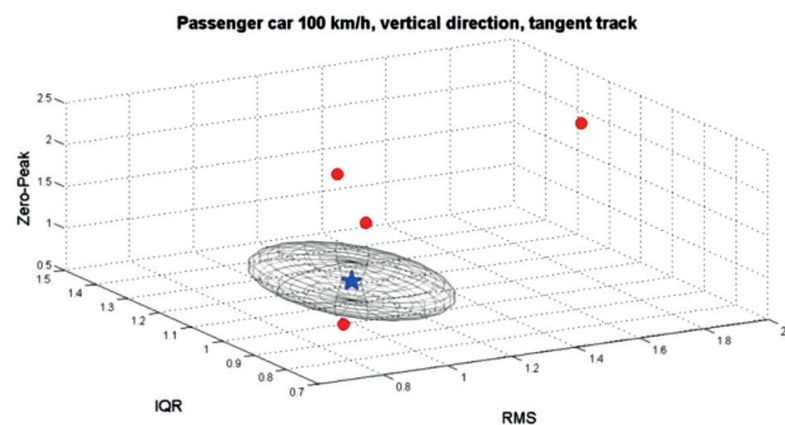

\begin{tabular}{|c|c|c|c|}
\hline RMS $(\mathrm{Z})$ & $\mathrm{IQR}(\mathrm{Z})$ & Zero-Peak $(\mathrm{Z})$ & $\mathrm{d}(\mathrm{Sv} . \mathrm{Ujv})$ \\
\hline 1.167 & 1.124 & 1.393 & 0.445 \\
\hline 1.389 & 1.438 & 1.317 & 0.666 \\
\hline 0.815 & 0.839 & 0.858 & 0.283 \\
\hline 1.787 & 1.073 & 2.328 & 1.546 \\
\hline
\end{tabular}

Fig. 6 Results of passenger car analysis, $100 \mathrm{~km} / \mathrm{h}$, vertical acceleration, tangent track

The results of the freight car condition analysis are depicted in Figs. 7 and 8. The results refer to a curved track section of $\mathrm{R}=900 \mathrm{~m}$. The reduction of stiffness to the half of its nominal value caused that majority of the points is located beyond boundaries of the reference point and clearly indicate damage. In case of the freight car number of passages was greater than for a passenger car, entailing more points in the plots. The radius $\mathrm{F}=0.144$ for $80 \mathrm{~km} / \mathrm{h}$ in lateral and $\mathrm{F}=0.112$ in vertical direction.

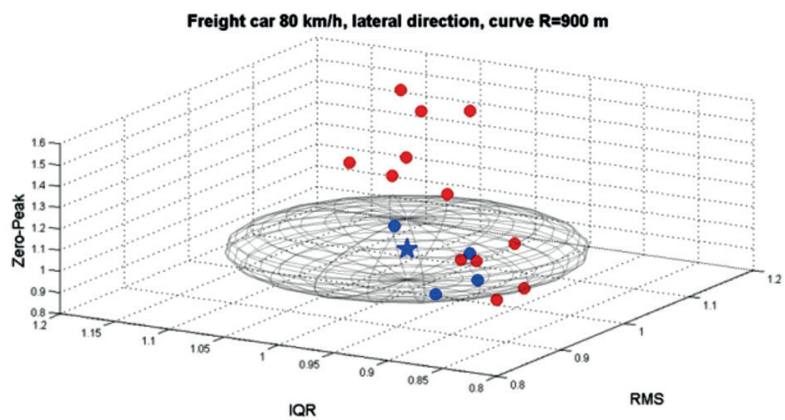

\begin{tabular}{|c|c|c|c|}
\hline RMS (Y) & IQR $(\mathrm{Y})$ & Zero-Peak $(\mathrm{Y})$ & $\mathrm{d}(\mathrm{Sv}, \mathrm{Ujv})$ \\
\hline 0.994 & 1.008 & 1.110 & 0.110 \\
\hline 1.069 & 0.983 & 1.575 & 0.580 \\
\hline 1.104 & 1.067 & 1.568 & 0.581 \\
\hline 1.185 & 1.096 & 1.345 & 0.403 \\
\hline 1.082 & 1.062 & 1.196 & 0.222 \\
\hline 0.960 & 0.878 & 1.165 & 0.209 \\
\hline 1.109 & 1.065 & 1.246 & 0.277 \\
\hline 1.054 & 0.995 & 1.194 & 0.201 \\
\hline 0.939 & 0.938 & 0.909 & 0.126 \\
\hline 0.934 & 0.879 & 0.933 & 0.154 \\
\hline 0.974 & 0.920 & 0.945 & 0.100 \\
\hline 0.972 & 0.926 & 1.068 & 0.105 \\
\hline 0.905 & 0.837 & 1.054 & 0.196 \\
\hline 0.894 & 0.874 & 1.169 & 0.236 \\
\hline 1.082 & 1.101 & 1.229 & 0.263 \\
\hline 0.924 & 0.906 & 1.114 & 0.166 \\
\hline
\end{tabular}

Fig. 7 Results of freight car analysis, $80 \mathrm{~km} / \mathrm{h}$, lateral acceleration, curve $R=900 \mathrm{~m}$

\section{Conclusions}

The method of multidimensional analysis of statistical parameters enables to detect faults of running gear (especially suspension). Vehicle condition is assessed on analysis of the metric between a reference point and a point representing current state. As the results have shown, it is necessary to take into consideration more points for the proper condition assessment. Although the vehicle may be damaged, some of the points might be located within permissible range (sphere) defined by $\mathrm{F}$ value. Setting the limit value of the metric should be preceded by statistical analysis of data acquired from nominal and damaged vehicle. For the experiment results analysis it was equal to standard deviation of distance from the points of current nominal condition to the reference point (averaged values for undamaged vehicle). In case of embracing too many points the $F$ value should be adjusted. 


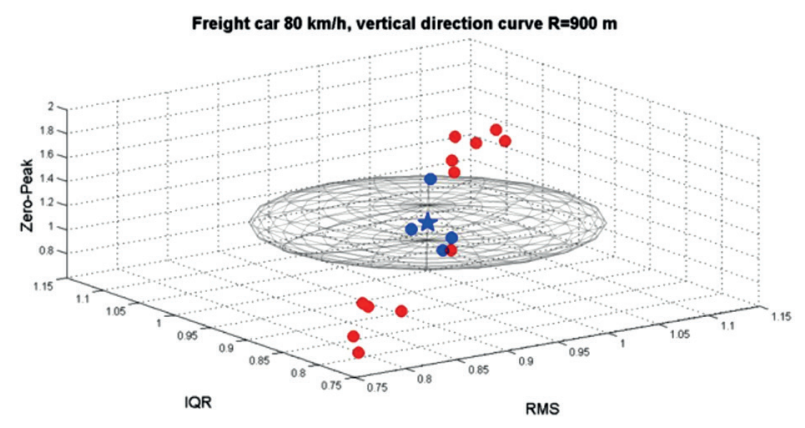

\begin{tabular}{|c|c|c|c|}
\hline RMS $(Z)$ & IQR $(Z)$ & Zero-Peak $(Z)$ & $\mathrm{d}(\mathrm{Sv}, \mathrm{Ujv})$ \\
\hline 0.832 & 0.847 & 0.866 & 0.264 \\
\hline 0.958 & 0.920 & 0.998 & 0.090 \\
\hline 1.137 & 1.125 & 1.201 & 0.273 \\
\hline 1.118 & 1.058 & 1.385 & 0.407 \\
\hline 0.919 & 0.854 & 1.193 & 0.255 \\
\hline 0.837 & 0.808 & 0.902 & 0.270 \\
\hline 1.122 & 1.133 & 1.258 & 0.315 \\
\hline 1.082 & 1.078 & 1.135 & 0.176 \\
\hline 0.793 & 0.800 & 1.050 & 0.292 \\
\hline 0.959 & 0.965 & 1.079 & 0.095 \\
\hline 1.077 & 1.013 & 1.631 & 0.636 \\
\hline 1.086 & 1.087 & 1.209 & 0.243 \\
\hline 0.779 & 0.793 & 0.809 & 0.358 \\
\hline 0.759 & 0.758 & 0.776 & 0.408 \\
\hline 1.062 & 1.083 & 1.099 & 0.144 \\
\hline 0.981 & 0.940 & 1.026 & 0.069 \\
\hline
\end{tabular}

Fig. 8 Results of freight car analysis, $80 \mathrm{~km} / \mathrm{h}$, vertical acceleration, curve $R=900 \mathrm{~m}$
On track sections of low maintenance quality the established limits $\mathrm{F}$ may cause generation of false alarms. It is necessary to observe the vehicle's location and relate the alarms with its current position on track sections.

The further work will include development of progressive limits $\mathrm{F}$ according to track quality indicator - calculated upon the basis of accelration signals obtained from axle boxes.

\section{Acknowledgement}

The authors express their gratitude for the financial support by the Polish Ministry of Science and Higher Education for the support of research within the framework of the project MONITORING OF TECHNICAL STATE OF CONSTRUCTION AND EVALUATION OF ITS LIFESPAN

- MONIT. Action Operational Programme Innovative Economy.

\section{References}

[1] GODA, K., GOODALL, R.: Fault-detection-and-isolation System for a Railway Vehicle Bogie. Vehicle System Dynamics Supplement 41 (2004), pp.468-476 Taylor \& Francis Ltd.

[2] LI, P., GOODALL, R.: Model-based Condition Monitoring for Railway Vehicle Systems, Control 2004, University of Bath, UK, September 2004

[3] TSUNASHIMA, H., et al.: Condition Monitoring and Fault Detection of Railway Vehicle Suspension using Multiple-Model Approach Proc. of the 17th World Congress, the Intern. Federation of Automatic Control, Seoul, 2008

[4] TSUNASHIMA, H., MORI, H.: Condition Monitoring of Railway Vehicle Suspension Using Adaptive Multiple Model Approach. Intern. Conference on Control, Automation and Systems 2010, Gyeonggi-do, Korea, 2010

[5] UIC 518: Testing and Approval of Railway Vehicles from the Point of View of Their Dynamic Behaviour - Safety - Track Fatigue - Ride Quality, 2003

[6] EN 14363: Railway applications - Testing for the Acceptance of Running Characteristics of Railway Vehicles - Testing of Running behaviour and Stationary Tests, 2005 


\section{COMMNICOIIONS}

[7] CHUDZIKIEWICZ, A., SOWINSKI, B.: Problems of Choosing Statistical Parameters In the Process of Monitoring the System of Railway Vehicle. Proc. of 8th Intern. Conference on Railway Bogies and Running Gears, pp.127-129, Hungary, Budapest, 8-10 November 2010

[8] CHUDZIKIEWICZ, A., SOWINSKI, B., SZULCZYK, A.: Statistical Parameters of Vibrations as Measures of Rail Vehicle Condition. Proc. of 17th Intern. Congress on Sound and Vibration, pp.73, Cairo, 2010

[9] MELNIK, R., KOSTRZEWSKI, M.: Rail Vehicle's Suspension Monitoring System - Analysis of Results Obtained From Tests of the Prototype. Key Engineering Materials, vol. 518, pp 281-288, 2012. 